\title{
Mediator redefines itself
}

Cell Research (2014) 24:775-776. doi:10.1038/cr.2014.76; published online 10 June 2014

Mediator is a large and structurally dynamic protein complex that is globally required for eukaryotic transcription by RNA polymerase II. In a recent paper published in Cell Research, Wang et al. report for the first time the location of distinct subunits and redefine domains in the S. cerevisiae Mediator complex.

The Mediator complex is specific to eukaryotic organisms and, despite significant divergence in subunit sequence identity, several basic functions of the complex (e.g., interaction with DNA-binding transcription factors and RNA polymerase II (pol II), and general requirement for genome-wide transcription ) are conserved between yeast and mammals [1]. Mediator possesses a disproportionately large number of predicted intrinsically disordered regions in the sequences of its subunits [2]. Many of the intrinsically disordered regions have sequence signatures that suggest the presence of protein-protein binding domains although the exact nature of these putative interactions remains largely unknown.

The first structures of the $S$. cerevisiae Mediator complex were resolved with electron microscopy (EM) [3]. Previous data revealed not only some conservation of structural features between yeast and mammalian Mediator complexes but also a great degree of flexibility and conformational dynamics $[3,4]$. For example, binding of pol II to the $S$. cerevisiae Mediator complex triggered extensive structural rearrangements.

The designations of the $S$. cerevisiae Mediator head, middle, and tail modules were made in part from EM 2D projections, based on the organization of protein density in the complex [3]. Understandably, however, a thorough confirmation of the location of specific subunits within the structure has not been completed. Given the large number of subunits, it would be a monumental task to systematically locate each Mediator subunit via EM labeling techniques. Whereas the study by Wang et al. does not label and localize each subunit in the Mediator complex, they combined EM labeling of four subunits with other strategies to reveal a modified architectural model of the $S$. cerevisiae Mediator complex [5]. As S. cerevisiae Mediator is structurally flexible and large in size (approximately $1 \mathrm{MDa} ; 21$ protein subunits), EM is a preferred technique for structural analysis of the complex. Although numerous EM studies have been completed for $S$. cerevisiae Mediator, the work presented by Wang et al. addresses some key issues and reveals some surprising and important insights.

A number of aspects of the study by Wang et al. stand out as being important for its success. First, a purification protocol was implemented that allowed isolation of yeast (S. cerevisiae) Mediator at high concentration $(1 \mathrm{mg} / \mathrm{ml})$. Second, the authors employed nanogold labeling strategies to enable localization of Med14, Med17, Med21 and Med22 within the complex. This represents the first time that specific subunits have been localized in EM maps of an entire yeast Mediator complex. Third, Wang et al. used a variety of approaches to isolate and structurally characterize the Mediator middle + head domains and middle domains only. This information helped validate and clarify the location of the middle, head and tail modules of the $S$. cerevisiae Mediator complex (Figure 1). The EM structural model for the middle module shown by Wang et $a l$. indicates an extended and apparently highly flexible shape, in agreement with published studies with recombinantly expressed subunits [6]. Taken together, the findings presented by Wang et al. provide new and very basic information about the subunit architecture of $S$. cerevisiae Mediator; the fact that such details remained uncertain reflects the challenges associated with structural analysis of the entire Mediator complex.

Importantly, the redefined structural organization of S. cerevisiae Mediator is consistent with existing genetic and biochemical studies. The Kornberg group, for example, has shown that the $S$. cerevisiae Mediator head module does not bind pol II on its own, but requires also the middle module and/or other preinitiation complex (PIC) components such as TFIIF [7]. These findings are supported by the architectural model proposed by Wang et al., as yeast pol II appears to interact predominantly with the head and middle domains based on the new model. Previous EM studies showed that "tail-less" S. cerevisiae Mediator complexes (i.e., containing only head + middle modules) could interact with the pol II enzyme [4]. Furthermore, $S$. pombe Mediator naturally lacks subunits that comprise the tail domain, like S. cerevisiae Mediator, and it also interacts with pol II [8]. The revised structural model is even consistent with possible structural roles for $S$. cerevisiae tail subunits in pol II-Mediator interactions during transcription [9].

Given the results from Wang et al., 


\section{Previous model}

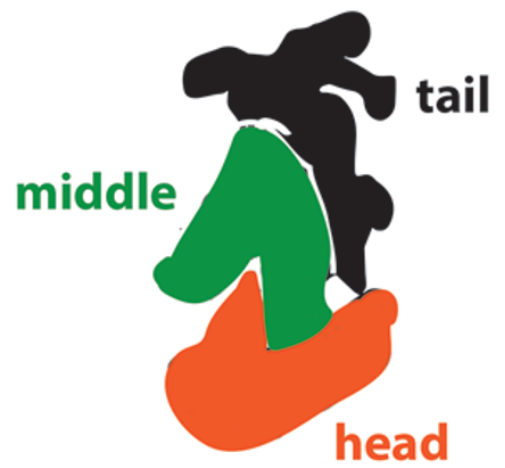

\section{Redefined model}

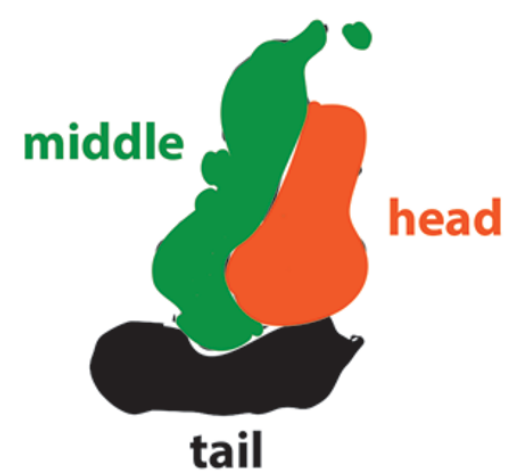

Figure 1 Schematic representation of a previous architectural model of $S$. cerevisiae Mediator (left) and the redefined model (right) based on data from the Cai group [5]. The S. cerevisiae Mediator complex contains 21 subunits: 7 in the head module (Med6, Med8, Med11, Med17, Med18, Med20, Med22), 9 in the middle module (Med1, Med4, Med7, Med9, Med10, Med14, Med19, Med21, Med31), and 5 in the tail module (Med2, Med3, Med5, Med15, Med16).

it will be interesting to see whether similar subunit organization is observed in mammalian Mediator complexes. Presently only a few subunits have been localized in the human Mediator complex, and this localization has been demonstrated indirectly via antibody labeling of transcription factors bound to the Mediator. Given the distinct subunit composition and poor sequence conservation, it is possible that Mediator subunit organization is distinct in mammals. It is also worth noting that the human Mediator complex undergoes structural changes that could significantly re-organize subunit architecture [1].

It remains to be determined how the head, middle, and tail modules of the $S$. cerevisiae Mediator complex re-organize upon binding to pol II and other components of the PIC. Initial EM studies of $S$. cerevisiae Mediator showed evidence for a dramatic structural re-organization upon interaction with pol II [3]. It is also unknown which or how many Mediator subunits directly interact with the pol II enzyme upon PIC assembly. Recent success with reconstitution and cryo-EM 3D reconstruction of the S. cerevisiae PIC lacking Mediator suggests that answers to these and other fundamental questions will soon be revealed [10].

Although the data presented by the Cai group [5] convincingly establish an updated structural model of the $S$. cerevisiae Mediator complex, it is noteworthy that the structural resolution is not better than previous studies. This is certainly not a reflection of the biochemical purification or the EM data, but rather results from the intrinsic flexibility and disorder of the Mediator complex [2]. Low-resolution structural data for the 21-subunit $S$. cerevisiae Mediator complex continues to limit understanding of its mechanism of action. Recent breakthroughs in EM instrumentation, such as direct electron detectors, and increasingly sophisticated image processing capabilities, offer great promise for improved understanding of this large, dynamic complex. Through the efforts of the Cai group and others, the complexities of Mediator structure and function will continue to be unraveled. Nicholas C Sennett
Dylan J Taatjes $^{1}$

${ }^{1}$ Department of Chemistry and Biochemistry, University of Colorado, Boulder, CO 80303, USA

Correspondence: Dylan J Taatjes

Tel: +1 303 492-6929; Fax: +1 303 492-5894

E-mail: Taatjes@colorado.edu

\section{References}

1 Poss ZC, Ebmeier CC, Taatjes DJ. Crit Rev Biochem Mol Biol 2013; 48:575-608.

2 Toth-Petroczy A, Oldfield CJ, Simon I, et al. PLoS Comput Biol 2008; 4:e1000243.

3 Asturias FJ, Jiang YW, Myers LC, et al. Science 1999; 283:985-987.

4 Dotson MR, Yuan CX, Roeder RG, et al. Proc Natl Acad Sci USA 2000; 97:1430714310 .

5 Wang X, Sun Q, Ding Z, et al. Cell Res 2014; 24:796-808.

6 Lariviere L, Plaschka C, Seizl M, et al. $\mathrm{Nu}$ cleic Acids Res 2013; 41:9266-9273.

7 Takagi Y, Calero G, Komori H, et al. Mol Cell 2006; 23:355-364.

8 Elmlund H, Baraznenok V, Lindahl M, et al. Proc Natl Acad Sci USA 2006; 103:1578815793.

9 Reeves WM, Hahn S. Mol Cell Biol 2003; 23:349-358.

10 Murakami K, Elmlund H, Kalisman N, et al. Science 2013; 342:1238724. 